\title{
Desafios na utilização do checklist de cirurgia segura
}

\author{
Challenges in using the safe surgery checklist
}

Desafíos en el uso de la lista de verificación de cirugía segura

\begin{abstract}
Vanessa Rodrigues da Silva ${ }^{1}$, Ruth Cardoso Rocha ${ }^{1}$, Manalde Ferreira da Silva ${ }^{1}$, Ingrid Moura de Abreu $^{1 *}$, Priscila Martins Mendes ${ }^{1}$, David Bernar Oliveira Guimarães ${ }^{1}$, Samya Raquel Soares Dias ${ }^{1}$, Maria do Carmo Santos Ferreira', Fernanda Valéria Silva Dantas Avelino'.
\end{abstract}

\section{RESUMO}

Objetivo: Avaliar a aplicação do checklist de cirurgia segura por profissionais de enfermagem. Métodos: Estudo descritivo transversal realizado no centro cirúrgico de um hospital universitário do nordeste, com 50 profissionais de enfermagem, no mês de novembro de 2016. Os dados foram coletados a partir da aplicação de questionário e analisados no programa IBM Statistical Package for the Social Sciences. Resultados: Dos 50 participantes do estudo, $66 \%$ responderam que "a aplicação do checklist promove melhora na qualidade do cuidado com benefícios para o paciente, profissional e instituição". No entanto, dentre as dificuldades/desvantagens apontadas, $30 \%$ dos sujeitos afirmaram que há "falta de colaboração da equipe em geral (não especificado)" representando obstáculo durante a sua utilização. Conclusão: Os profissionais demonstram conhecimento da existência e da importância da aplicação do checklist, mas encontram dificuldades na sua utilização.

Palavras-chave: Lista de checagem, Enfermagem Perioperatória, Segurança do paciente.

\begin{abstract}
Objective: To evaluate the application of the safe surgery checklist by nursing professionals. Methods: A descriptive, cross-sectional study carried out in the surgical center of a university hospital in northeast, with 50 nursing professionals, in November 2016. Data were collected from the questionnaire application and analyzed in the IBM Statistical Package for the Social Sciences program. The data were analyzed by descriptive statistics using IBM SPSS Statistics software. Results: Of the 50 participants in the study, $66 \%$ answered that "the application of the checklist promotes improvement in the quality of care with benefits for the patient, professional and institution". However, among the difficulties/ disadvantages pointed out, $30 \%$ of the subjects stated that there is "lack of collaboration of the general team (unspecified)" representing an obstacle during their use. Conclusion: Professionals demonstrate knowledge of the existence and importance of applying the checklist, but find difficulties in its use.
\end{abstract}

Key words: Checklist, Perioperative Nursing, Patient Safety.

RESUMEN

Objetivo: Evaluar la aplicación de la lista de verificación de cirugía segura por profesionales de enfermería. Métodos: un estudio descriptivo y transversal realizado en el centro quirúrgico de un hospital universitario in noreste, con 50 profesionales de enfermería, en noviembre de 2016. Los datos se recopilaron de la aplicación del cuestionario y se analizaron en el Paquete Estadístico de IBM para el programa de Ciencias Sociales. Resultados: De los 50 participantes en el estudio, el 66\% respondió que "la aplicación de la lista de verificación promueve el mejoramiento de la calidad de la atención con beneficios para el paciente, el profesional y la institución". Sin embargo, entre las dificultades / desventajas señaladas, el $30 \%$ de los sujetos afirmaron que existe una "falta de colaboración del equipo general (no especificado)" que representa un obstáculo durante su uso. Conclusión: los profesionales demuestran el conocimiento de la existencia y la importancia de aplicar la lista de verificación, pero encuentran dificultades en su uso.

Palabras clave: Lista de Verificación, Enfermería Perioperatoria, Seguridad del Paciente.

1 Universidade Federal do Piauí- UFPI, Teresina (Piauí). *E-mail: ingridmabreu@outlook.com

SUBMETIDO EM: 8/2019 ｜ＡCEITO EM: 9/2019｜ＰULICADO EM: 9/2019

REAS/EJCH | Vol.11(16) | e1472 | DOI: https://doi.org/10.25248/reas.e1472.2019 Página 1 de 8 


\section{INTRODUÇÃO}

Um dos setores da saúde em que frequentemente acontecem incidentes que provocam danos ao paciente, também chamados de eventos adversos (EA) é o centro cirúrgico (CC), apresentando causas multifatoriais e muitas vezes atribuída a complexidade dos procedimentos, a necessidade da interação de equipes interdisciplinares e ao trabalho sob pressão. Os riscos ao paciente são uma realidade presente na assistência cirúrgica e compete às equipes envolvidas no processo de cuidado propor estratégias e estabelecer barreiras para garantir a segurança do paciente (MANRIQUE BT, et al., 2015).

A segurança do paciente é definida como a ausência de danos ou de lesões acidentais durante a prestação de assistência à saúde (PIRES MPO, PEDREIRA MLG e PETERLINE MAS, 2013). Diante disso, acredita-se que o gerenciamento de risco possibilita às equipes avaliarem o cuidado oferecido ao paciente no CC, observando e propondo melhores práticas que minimizem problemas ou até mesmo antecipando-os frente aos riscos (GOMES CDPP, et al., 2016).

No intuito de se reduzir esses riscos, a Organização Mundial da Saúde (OMS), em 2008, lançou a campanha "Cirurgias Seguras Salvam Vidas" que faz parte do Segundo Desafio Global para a Segurança do Paciente. Juntamente com a campanha e em parceira com a Agência Nacional de Vigilância Sanitária (ANVISA), a OMS lançou um manual que contém a Lista de Verificação de Segurança Cirúrgica (checklist), proposta para ser empregada em qualquer hospital, cujo objetivo é auxiliar as equipes cirúrgicas a seguirem de forma sistemática passos críticos de segurança (BRASIL, 2009).

O checklist proposto pela OMS inclui 19 itens divididos em três momentos críticos do procedimento cirúrgico: antes da indução anestésica (fase 1), antes da incisão cutânea (fase 2) e antes do paciente sair da sala de operação (fase 3). Todas as etapas devem ser checadas verbalmente com as pessoas envolvidas no processo para assegurar que as ações foram realizadas (TORRES-MANRIQUE B, et al., 2016).

Cerca de um em cada cinco pacientes que sofrem EA cirúrgicos evoluem para incapacidade permanente ou morte. Diante dessa realidade, o checklist é capaz de reduzir as taxas de mortalidade e de complicações, aumentar a adesão à antibioticoprofilaxia e reduzir o número de erros por falha de comunicação da equipe favorecendo a redução de problemas (FREITAS MR, et al, 2014).

Apesar dos benefícios comprovados, sua integração à prática clínica na área da saúde no Brasil ainda não se faz de forma tão rápida e ampla como em outros campos. Nessa perspectiva tornou-se necessário responder às seguintes perguntas: quais as facilidades/vantagens e dificuldades/desvantagens da utilização do checklist? Quais os erros associados ao preenchimento pela equipe de enfermagem? Para responder os questionamentos o presente estudo objetivou avaliar a aplicação do checklist por profissionais de enfermagem.

\section{MÉTODOS}

Trata-se de um estudo descritivo e transversal realizado no CC de um Hospital Universitário do nordeste brasileiro. A população estudada foi composta pelos profissionais da equipe de enfermagem, a amostra foi não probabilística, por conveniência, totalizando 50 participantes na pesquisa, sendo 9 enfermeiros e 41 técnicos de enfermagem. Foram incluídos os profissionais que trabalhassem há no mínimo seis meses no $\mathrm{CC}$, foram excluídos os que estavam de férias ou licença.

Para a obtenção dos dados optou-se pela aplicação de um questionário elaborado pelos próprios pesquisadores, composto por 20 perguntas, destas 18 objetivas e 2 subjetivas. O questionário é constituído de questões com variáveis sociodemográficas e questões que avaliam o uso do checklist no CC.

Os dados foram coletados durante do mês de novembro de 2016, por meio da abordagem consentida e aplicação de questionário no próprio local de trabalho em horário disponível do participante.

Os dados foram armazenados em planilha do Microsoft Excel e as análises estatísticas realizadas por meio do programa IBM Statistical Package for the Social Sciences (SPSS) versão 20.0. Foi feito um estudo 
estatístico descritivo analítico da amostra através das frequências absolutas ( $n$ ) e relativas (\%), medidas de tendência central (mínimo e máximo) e medidas de dispersão (média e desvio padrão), usando o teste Quiquadrado de Aderência para verificar a diferença estatística entre as variáveis. $O$ nível de significância adotado foi de $\alpha=0,05$. Os resultados estão apresentados em tabelas e gráficos.

O projeto de pesquisa foi aprovado pelo Comitê de Ética em Pesquisa com parecer no 1.755 .815 e CAAE: 50273715.1.0000.5214. Aos participantes foi apresentado o Termo de Consentimento Livre e Esclarecido (TCLE), garantindo a confidencialidade e privacidade, conforme os princípios dispostos na Resolução no 466/2012 do Conselho Nacional de Saúde

\section{RESULTADOS}

O perfil sociodemográfico dos participantes da pesquisa mostrou que $92 \%$ era do sexo feminino, $28 \%$ apresentava idade entre 25 e 30 anos e $38 \%$ possuía grau de instrução equivalente a pós-graduação (especialização). Quanto ao cargo/função no hospital, 18\% eram enfermeiros e $82 \%$ técnicos de enfermagem. Em relação ao perfil de trabalho da equipe de enfermagem, $86 \%$ possuía um tempo de trabalho de 1 a 3 anos no hospital. Todos os participantes trabalhavam de 20 a 39h por semana. Em relação ao tempo de trabalho na profissão atual, $94 \%$ do total de profissionais responderam à pergunta e destes, $57,4 \%$ declararam ter entre 5 e 10 anos de atuação.

Sobre a sua aplicação, quando questionados se conheciam o checklist de cirurgia segura $98 \%$ dos participantes responderam "sim", $80 \%$ disseram já ter recebido treinamento para sua aplicação, 98\% já utilizou/preencheu em sua vida profissional, $98 \%$ afirmou que gostaria que o checklist fosse aplicado em uma sala cirúrgica caso estivesse atuando, $66 \%$ observou mudanças na comunicação interpessoal da equipe com a sua utilização, 100\% dos participantes julga passível a aplicação na instituição (Figura 1).

Figura 1 - Aplicação do checklist de Cirurgia Segura em pacientes cirúrgicos de um Hospital Universitário, Teresina - PI, 2016.

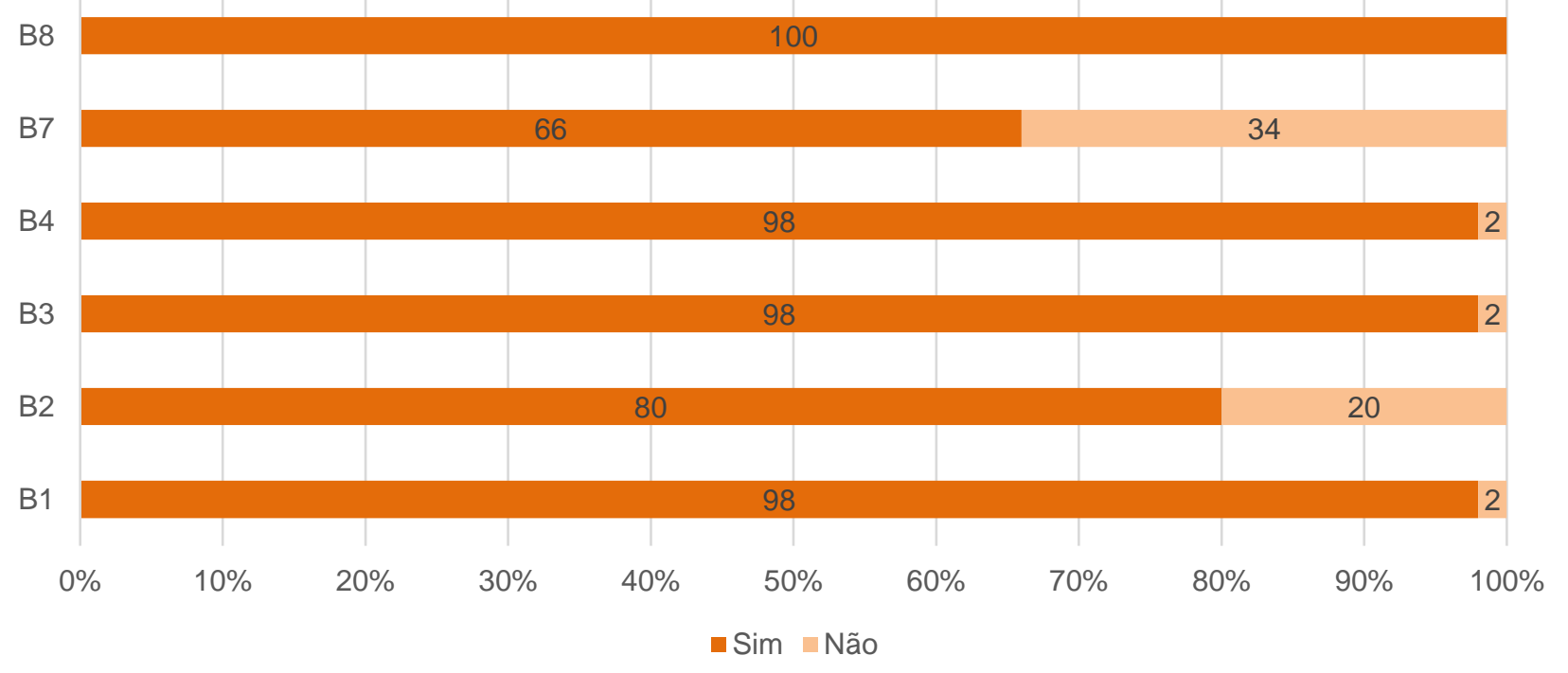

Legenda: B1 - Você já conhece algum checklist sobre segurança em sala de cirurgia?; B2 - Você já recebeu treinamento para aplicar o checklist; B3 - Você, em algum momento de sua atividade profissional, já utilizou/preencheu o checklist?; B4 - Se você estivesse atuando em uma centro cirúrgico cirúrgica, você acharia interessante que o checklist fosse aplicado?; B7 - Você notou mudanças na comunicação da equipe cirúrgica com a aplicação do checklist?; B8 - Você considera viável a aplicação deste checklist de segurança cirúrgica nesta instituição?

Fonte: Silva VR, Rocha RC, Silva MF, et al., 2016. 
Em relação às facilidades/vantagens do uso do checklist, pelo teste qui-quadrado de aderência, observase que houve significância estatística $(p<0,05)$ no item "o instrumento proporciona segurança ao paciente e a equipe, prevenindo erros" (68\%). Outra parcela (66\%) respondeu que "a sua aplicação promove melhora na qualidade do cuidado com benefícios para o paciente, profissional e instituição".

No que diz respeito as dificuldades/desvantagens, os resultados demonstraram que $32,6 \%$ dos profissionais relataram "Falta tempo para o preenchimento". Ainda houve um número expressivo (69,8\%) no item do questionário "Outra" em relação aos demais, com grau de significância estatístico $(p<0,05)$. Como essa alternativa tratava-se de uma questão aberta, foi necessária a categorização dos dados de acordo com as justificativas: "falta de colaboração da equipe médica", "falta de colaboração da equipe em geral (não especificado)", "não apresenta dificuldade/desvantagem", "alguns profissionais se recusam a responder" e "não especificado". Desta forma, $36,7 \%$ responderam que o uso do checklist não apresentava dificuldade/desvantagem, apesar disso, $30 \%$ afirmaram que a 'falta de colaboração da equipe em geral (não especificado)' é uma dificuldade durante o uso do checklist (Tabela 1).

Tabela 1 - Facilidades/Vantagens e Dificuldades/Desvantagens do uso do checklist nas cirurgias em um Hospital Universitário, Teresina - PI, 2016.

\begin{tabular}{|c|c|c|c|}
\hline & $\mathbf{N}$ & $\%$ & p-valor ${ }^{1}$ \\
\hline \multicolumn{4}{|l|}{ Facilidades/Vantagens } \\
\hline $\begin{array}{l}\text { O instrumento proporciona segurança ao paciente e à equipe, } \\
\text { prevenindo erros }\end{array}$ & 34 & 68,0 & $<0,001^{*}$ \\
\hline O preenchimento é realizado com rapidez e praticidade & 11 & 22,0 & \\
\hline $\begin{array}{l}\text { A aplicação do checklist promove melhora na qualidade do } \\
\text { cuidado com benefícios para o paciente, profissional e instituição }\end{array}$ & 33 & 66,0 & \\
\hline Outra & 2 & 4,0 & \\
\hline \multicolumn{4}{|l|}{ Dificuldades/Desvantagens } \\
\hline Falta tempo para o preenchimento & 14 & 32,6 & \\
\hline Aumenta a sobrecarga de trabalho & 1 & 2,3 & \\
\hline $\begin{array}{l}\text { Não proporciona segurança à equipe, apenas contribuindo para } \\
\text { aumentar a burocracia existente dentro do centro cirúrgico }\end{array}$ & - & - & \\
\hline Outra & 30 & 69,8 & $<0,001^{*}$ \\
\hline Falta de colaboração da equipe médica & 4 & 13,3 & \\
\hline Falta de colaboração da equipe em geral (não especificado) & 9 & 30,0 & \\
\hline Não apresenta dificuldade/desvantagem & 11 & 36,7 & \\
\hline Alguns profissionais se recusam a responder & 2 & 6,7 & \\
\hline Não especificado & 4 & 13,3 & \\
\hline
\end{tabular}

\#Resposta múltipla. Percentual ultrapassa os 100\%. ${ }^{1}$ Teste Qui-quadrado de Aderência; *Significativo ao nível de $5 \%$.

Fonte: Silva VR, Rocha RC, Silva MF, et al., 2016.

A seguir observa-se a avaliação do uso do checklist pela equipe de enfermagem, conforme cargo profissional na unidade de trabalho. Verificou-se significância estatística entre os cargos de enfermeiro e técnico em enfermagem nas classificações bom e regular $(p<0,05)$. Destacando-se que $50 \%$ dos profissionais avaliaram o uso do checklist como "bom", houve maior significância estatística no cargo de técnico em enfermagem (53,7\%) (Tabela 2). 
Tabela 2 - Avaliação do uso do checklist pela equipe de enfermagem no Centro Cirúrgico de um Hospital Universitário, Teresina - PI, 2016.

\begin{tabular}{lcccc}
\hline & \multicolumn{2}{c}{ Cargo/Função } & & \\
\cline { 2 - 4 } & Enfermeiro & $\begin{array}{c}\text { Técnico de } \\
\text { enfermagem }\end{array}$ & Total & p-valor ${ }^{1}$ \\
\cline { 2 - 4 } & $\mathbf{n ( \% )}$ & $\mathbf{n}(\%)$ & $\mathbf{n}(\%)$ & \\
\hline Excelente & $2(22,2)$ & $4(9,8)$ & $6(12,0)$ & 0,414 \\
Bom & $3(33,4)$ & $22(53,7)$ & $25(50,0)$ & $<0,001^{*}$ \\
Regular & $2(22,2)$ & $11(26,8)$ & $13(26,0)$ & $0,012^{*}$ \\
Ruim & $2(22,2)$ & $3(7,3)$ & $5(10,0)$ & 0,654 \\
Muito ruim & - & $1(2,4)$ & $1(2,0)$ & 0,317 \\
Total & $9(100,0)$ & $41(100,0)$ & $50(100,0)$ & -
\end{tabular}

TTeste Qui-quadrado de Aderência; *Significativo ao nível de 5\%.

Fonte: Silva VR, Rocha RC, Silva MF, et al., 2016.

Verificou-se, ainda, os principais erros associados ao preenchimento do checklist. $A$ maioria dos profissionais $(36 \%)$ classificou como rara a ocorrência de erros associados ao preenchimento. Entre os motivos de falhas apontados pelos profissionais, destacaram-se a falta de compromisso/interesse da equipe com $36 \%(p<0,01)$ e a falta de tempo $(14 \%)$. Quanto às complicações por erros no preenchimento, $72 \%$ dos profissionais declararam não ter ocorrido e 16\% apontou para a ocorrência de complicações consequentes a falhas no preenchimento; entre elas destacaram-se problemas nos equipamentos da sala de cirurgia, troca de procedimento e troca de pacientes (Tabela 3).

Tabela 3 - Erros associados ao preenchimento do checklist de um Hospital Universitário, Teresina - PI, 2016.

\begin{tabular}{lccc}
\hline Variáveis & N & $\%$ & p-valor $^{1}$ \\
\hline Frequência de erros no preenchimento do checklist & & & \\
\hline Nunca observei & 8 & 16,0 & 0,193 \\
Raramente & 18 & 36,0 & \\
Esporadicamente & 14 & 28,0 & \\
Frequentemente & 10 & 20,0 & \\
\hline Principal motivo que leva à falha no preenchimento & & & \\
\hline Falta de compromisso/interesse da equipe (não especificado) & 18 & 36,0 & $<0,001^{*}$ \\
Falta de compromisso/interesse da equipe médica & 3 & 6,0 & \\
Falta de atenção & 6 & 12,0 & \\
Falha na comunicação & 3 & 6,0 & \\
Falta de tempo & 7 & 14,0 & \\
Falta de conhecimento/treinamento da equipe & 3 & 6,0 & \\
Pressa da equipe médica & 6 & 12,0 & \\
Urgências e cirurgias não programadas & 1 & 2,0 & \\
Recusa da equipe (não especificado) & 0 & 0,0 & \\
Não respondeu & 3 & 6,0 & \\
Complicação ocasionada pelo erro de preenchimento & \multicolumn{2}{c}{$<0,001^{*}$} \\
Não & 36 & 72,0 & \\
Sim & 8 & 16,0 & \\
Não respondeu & 6 & 12,0 & \\
\hline
\end{tabular}

TTeste Qui-quadrado de Aderência; *Significativo ao nível de 5\%.

Fonte: Silva VR, Rocha RC, Silva MF, et al., 2016. 


\section{DISCUSSÃO}

Observou-se uma predominância feminina, que segundo pesquisa realizada em São Paulo, é a reprodução de uma característica histórica da enfermagem, profissão que vem sendo exercida em sua maioria por mulheres (MARTINS C, et al., 2006). Além disso, esse aspecto é considerado um traço de muitas culturas onde há força do trabalho feminino em atividades que envolvam o cuidado com pessoas.

Em relação à idade, neste $\mathrm{CC}$ encontrou-se um perfil relativamente jovem variando em sua maioria de 25 a 35 anos. Os profissionais de enfermagem que se encontram nessa faixa etária merecem uma atenção maior do gestor, já que precisam aprender a lidar com as demandas desse ambiente de trabalho. Em decorrência disto e da necessidade de desenvolver autonomia e responsabilidade, esses trabalhadores podem apresentar maiores níveis de estresse (SANTANA JCB, et al., 2015).

Em relação ao maior grau de instrução, observou-se uma predominância de profissionais especializados. Esse aspecto é esperado, uma vez que, a instituição tratava-se de um hospital de ensino e além de investimentos em pesquisa e extensão, a contratação de profissionais qualificados é de fundamental importância para uma adequada assistência. O tempo de trabalho no hospital e o tempo de trabalho na atual área/unidade do hospital em estudo são relativamente recentes já que se trata de uma instituição de saúde que iniciou suas atividades em 2012, objetivando a prestação de assistência à saúde e a realização de atividades de ensino, pesquisa, extensão e inovação tecnológica (SANTANA JCB, et al., 2015).

Toda a amostra analisada trabalha de 20 a 39 horas por semana. De acordo com estudo que visou discorrer sobre a regulação das relações de trabalho na enfermagem, a carga horária semanal de trabalho varia de trinta a quarenta horas semanais, sendo mais comum a jornada de trinta e seis horas/semana (FREITAS GF, FUGULIN FMT e FERNANDES MFP, 2006).

Conforme os resultados da presente pesquisa, boa parte dos participantes mostrou familiaridade e conhecimento acerca da utilização do checklist. Dessa forma, entendem que cuidados simples como a conferência dos dados do paciente, informações clínicas, disponibilidade e funcionamento adequado de recursos materiais podem fazer a diferença em um procedimento, resultando em sucesso ou fracasso. (PANCIERI AP, et al., 2013). Segundo estudo realizado em sete países com o objetivo de comprovar a hipótese de que a implementação de checklist associada a mudanças culturais reduziria as taxas de óbito e complicações pós-cirurgia, $84 \%$ dos sujeitos onde foi implantado, relataram também uma melhora de comunicação na sala cirúrgica (HAYINES AB, et al, 2009).

Quando há uma falha nesse processo, as chances de ocorrerem erros médicos e EA é alta, pois não existe transferência de informações. Segundo pesquisa realizada em um hospital escola no estado de São Paulo Pancieri AP, et al., (2013), o uso adequado dessa ferramenta é facilitado quando as equipes passam a reconhecer a importância de inseri-lo à prática diária, já que provoca diminuição das divergências entre a equipe cirúrgica em situações inesperadas.

Um cuidado mais humanizado, que evita situações de descontinuidade da assistência e risco para o paciente é caracterizado por uma equipe coordenada, com boa comunicação interpessoal e que trabalha, principalmente, de forma cooperativa. A valorização de uma cultura organizacional que priorize esses aspectos é fundamental para a consolidação das estratégias para melhoria da qualidade da assistência e segurança do paciente em um CC (CARVALHO PA, et al., 2015).

A análise dos dados permitiu verificar ainda que a função desempenhada pelo profissional na unidade de trabalho tem correlação positiva com a percepção deste a respeito da utilização do checklist, apresentandose estatisticamente significante entre enfermeiros e técnicos de enfermagem quando o instrumento é classificado como "bom" ou "regular". A melhor avaliação feita pelos técnicos pode justificar-se pela quantidade de entrevistados e pelo fato dessa classe de profissionais possuir maior proximidade com 0 instrumento, visto que são eles que o preenchem na maioria das vezes. Considerando que a equipe de enfermagem participa do procedimento cirúrgico em suas diversas etapas, o checklist constitui importante documento de registro de ações indispensáveis à segurança (MAZIERO ECS, et al, 2015). 
A associação positiva entre os erros relacionados ao preenchimento do checklist e os motivos e complicações associadas às falhas no preenchimento denota negligência dos profissionais quanto ao registro e verificação de informações e a necessidade de desenvolver estratégias que promovam cultura de segurança em todos os níveis de profissionais de saúde, sejam assistenciais ou líderes e gerentes, quanto à sua importância (AMAYA MR, et al., 2015).

Erro nenhum pode ser considerado insignificante, independente de quem seja a responsabilidade, a principal forma de evitá-lo é identificar antecipadamente os fatores que podem desencadeá-lo e o checklist é um instrumento com esta finalidade. Quanto aos motivos de falha no preenchimento do checklist a falta de compromisso destaca-se como principal fator apontado pelos profissionais, seguido da falta de tempo, falta de atenção e pressa da equipe médica; fatores também indicados em outros estudos realizados na região sul e nordeste do Brasil (FREITAS MR, et al, 2014; AMAYA MR, et al, 2015).

A implementação do checklist, importante ferramenta na comunicação entre a equipe cirúrgica, se configura como um processo complexo partindo de um ponto de vista administrativo. A resistência dos profissionais às mudanças e a adaptação da lista às necessidades do ambiente e da especificidade no local são duas circunstâncias que devem ser consideradas para haver um resultado eficaz. Dentre outros desafios que podem ser encontrados destacam-se a dificuldade da equipe de enfermagem em entender a importância da realização da checagem nos momentos preconizados pela OMS e a dificuldade de preenchimento de alguns itens presentes no instrumento (PANCIERI A, CARVALHO R e BRAGA E, 2014).

Os registros conferem segurança para o profissional, esclarecendo as condições sob as quais os serviços de saúde foram prestados e além disso, auxiliam na execução de práticas mais seguras. Conforme pesquisa realizada em Murcia, na Espanha, a instituição do checklist nos procedimentos realizados em ambiente hospitalar reduz as taxas de mortalidade e complicações, aumenta a adesão à antibioticoprofilaxia e reduz 0 número de erros por falha de comunicação da equipe (ALEDO VS, et al., 2012).

O estudo limitou-se a uma única instituição o que reduz a caracterização do perfil local. Portanto, sugerese em pesquisas futuras a avaliação ampliada da adesão ao checklist em mais instituições. As contribuições do trabalho voltam-se à educação e conscientização de profissionais, favorecendo o processo de correto preenchimento do checklist de cirurgia segura, objetivando, assim, melhora na assistência e gestão do trabalho. Dessa maneira, torna-se fundamental promover a reflexão e o debate sobre a relevância desse instrumento para a qualidade da assistência, sobre os fatores que dificultam a sua utilização correta e ainda os recursos que podem facilitá-la.

\section{CONCLUSÃO}

O estudo possibilitou uma avaliação geral da aplicação do checklist por profissionais de enfermagem do CC revelando que embora esses profissionais tenham conhecimento da existência e da importância do checklist e que a sua aplicação promove melhora na qualidade do cuidado com benefícios para o paciente, profissional e instituição, ainda encontram dificuldades para sua utilização, principalmente no que diz respeito a falta de tempo e falta de colaboração/interesse da equipe. As informações obtidas neste estudo apontam ainda para as necessidades de melhora na aplicação do instrumento por parte da instituição, permitindo que as equipes cirúrgicas desenvolvam suas atividades de forma mais segura, comunicativa, produtiva e eficaz.

\section{REFERÊNCIAS}

1. ALEDO VS, et al. Dificultades en la implantación del checklist en los quirófanos de cirugía. Cir Esp. [Internet] 2012; 180(5): 180-185.

2. AMAYA MR, et al. Análise do registro e conteúdo de checklists para cirurgia segura. Esc. Anna Nery. [Internet] 2015; 19(2): 246-251.

3. BRASIL. Ministério da Saúde. Segundo desafio global para a segurança do paciente: Cirurgias seguras salvam vidas. Rio de Janeiro: Organização Pan-Americana da Saúde; Ministério da Saúde; Agência Nacional de Vigilância Sanitária; 2009. 
4. CARVALHO PA, et al. Cultura de segurança no centro cirúrgico de um hospital público, na percepção dos profissionais de saúde. Rev. Latino-Am. Enfermagem. [Internet] 2015; 23(6): 1041-1048.

5. FREITAS GF, FUGULIN FMT, FERNANDES MFP. A regulação das relações de trabalho e o gerenciamento de recursos humanos em enfermagem. Rev. Esc. Enferm. USP [Internet]. 2006; 40(3): 434-438.

6. FREITAS MR, et al. Avaliação da adesão ao checklist de cirurgia segura da OMS em cirurgias urológicas e ginecológicas, em dois hospitais de ensino de Natal, Rio Grande do Norte, Brasil. Cad. Saúde Pública [Internet]. $2014 ; 30(1): 137-148$.

7. GOMES CDPP, et al. Percepção de uma equipe de enfermagem sobre a utilização do checklist cirúrgico. Revista SOBECC [Internet]. 2016; 21(3): 140-145.

8. HAYNES AB, et al. A surgical safety checklist to reduce morbity and mortality in a global population. $\mathrm{N}$ Engl $\mathrm{J}$ Med. [Internet] 2009; 360(5): 491-499.

9. MANRIQUE BT, et al. Segurança do paciente no centro cirúrgico e qualidade documental relacionadas à infecção cirúrgica e à hospitalização. Acta paul. enferm. [Internet]. 2015; 28( 4 ): 355-360.

10. MARTINS C, et al. Perfil do enfermeiro e necessidades de desenvolvimento de competência profissional. Rev. Texto Contexto Enferm. 2006; 15(3):472-478.

11. MAZIERO ECS, et al. Adesão ao uso de um checklist cirúrgico para segurança do paciente. Rev Gaúcha de Enferm. [Internet] 2015;36(4): 14-20.

12. PANCIERI A, CARVALHO R, BRAGA E. Aplicação do checklist para cirurgia segura: Relato de experiência. Revista SOBECC [Internet]. 2014 Mar; 19(1): 26-33.

13. PANCIERI AP, et al. Checklist de cirurgia segura: análise da segurança e comunicação das equipes de um hospital escola. Rev Gaúcha Enferm. [Internet] 2013; 34(1): 71-78.

14. PIRES MPO, PEDREIRA MLG, PETERLINI MAS. Cirurgia segura em pediatria: elaboração e validação de checklist de intervenções pré-operatórias. Rev. Latino-Am. Enfermagem [Internet]. 2013; 21(5): 1-8.

15. SANTANA JCB, et al. Perfil dos técnicos de enfermagem de um serviço de atendimento pré-hospitalar. Rev. Enfermagem Revista [Internet]. 2015; 18(1): 16-27.

16. TORRES-MANRIQUE B, et al. Análisis cultural de los ítems de dos listas de verificación quirúrgica de España y Argentina. Rev. Gaúcha Enferm. [Internet]. 2016; 37( 3 ): e56359. 